\title{
Comet dust as a mixture of aggregates and solid particles: model consistent with ground-based and space-mission results
}

\author{
Ludmilla Kolokolova $^{1}$ and Hiroshi Kimura ${ }^{2}$ \\ ${ }^{1}$ Department of Astronomy, University of Maryland, College Park, MD, 20740, USA \\ ${ }^{2}$ Institute of Low Temperature Science, Hokkaido University, Sapporo 060-0819, Japan
}

(Received July 24, 2008; Revised December 8, 2008; Accepted December 8, 2008; Online published February 12, 2010)

\begin{abstract}
The most successful model of comet dust presents comet particles as aggregates of submicron grains. It qualitatively explains the spectral and angular change in the comet brightness and polarization and is consistent with the thermal infrared data and composition of the comet dust obtained in situ for comet 1P/Halley. However, it experiences some difficulties in providing a quantitative fit to the observational data. Here we present a model that considers comet dust as a mixture of aggregates and compact particles. The model is based on the Giotto and Stardust mission findings that both aggregates (made mainly of organics, silicates, and carbon) and solid silicate particles are present in the comet dust. We simulate aggregates as Ballistic Cluster-Cluster Aggregates (BCCA) and compact particles as polydisperse spheroids with some distribution of the aspect ratio. The particles follow a power-law size distribution with the power -3 that is close to the one obtained for comet dust in situ, at studies of the Stardust returned samples, and the results of ground-based observations of comets. The model provides a good fit to the angular polarization curve. It also reproduces the positive spectral gradient of polarization, red color of the dust, and low albedo. It also has the ratio of compact to fluffy particles close to the one found in situ for comet 1P/Halley and the mass ratio of silicate to carbonaceous materials equal to unity that is in accordance with the elemental abundances of Halley's dust found by Giotto mission.
\end{abstract}

Key words: Comet, dust, polarization, aggregates, silicate, Stardust, Giotto.

\section{Introduction}

Comets are bodies that have preserved the pristine materials from the time of the planet formation. Thus, knowledge about the comet dust allows us to find out the properties of the materials in the early Solar System. This is why comets have been objects of special interest not only to ground-based observers but also to numerous space missions: Giotto and Rosetta (European Space Agency), Vega (Soviet Space Agency), Suisei (Institute of Space and Astronautical Science, currently a section of Japanese Aerospace Exploration Agency), and NASA missions Contour, DS1, Stardust, and Deep Impact.

Accumulation of the observational data about comet dust (i.e., observations in the comet continuum filters free of gas emissions) reveals how comet dust brightness and polarization change with the phase angle (angle Sun-CometObserver) and with the wavelength in the visible and near-infrared spectral range (see details in the review by Kolokolova et al., 2004a). There were numerous attempts to use these data to solve the inverse problem for comet dust, i.e., to find out size, composition, shape, and structure of its particles based on the observational data. Unfortunately, the interpretation usually was done based only on photometric or only polarimetric data or was limited only by the angular

Copyright (C) The Society of Geomagnetism and Earth, Planetary and Space Sciences (SGEPSS); The Seismological Society of Japan; The Volcanological Society of Japan; The Geodetic Society of Japan; The Japanese Society for Planetary Sciences; TERRAPUB.

doi:10.5047/eps.2008.12.001 dependencies of the observed characteristics. In such a limited case, one could find particles (sometimes rather simple, e.g., spheres or cylinders) whose light-scattering properties fit some observational data. However, any attempts to use the same model of comet dust to interpret other observational data failed (see Kolokolova et al., 2004a for review and references). It was shown in Kolokolova et al. (2004b) that as soon as we try to fit photometric and polarimetric angular and spectral data together, no model of compact particles can be applied. Even the most complex model of polydisperse multishaped particles could not fit the whole scope of the observational data no matter what refractive index was tried. Even considering the comet particles as porous by calculating their refractive index using Maxwell Garnett mixing rule and considering voids as part of the mixture (similar to the approach described in Mukai et al., 1992) no ensemble of particles that could reproduce the correct shape of polarization and brightness phase curve together with the correct color and polarimetric spectral gradient (polarimetric color) was found.

Recently, a significant progress has been achieved in explaining the observational data describing the comet dust as an ensemble of aggregates made of submicron particles (see Kimura et al., 2003, 2006; Mann et al., 2004). This model allows not only to qualitatively reproduce the observed changes in brightness and polarization with phase angle and wavelength but also can explain existence of two types of comet infrared spectra characterized by strong and weak $10 \mu \mathrm{m}$ silicate feature. It was shown in Kolokolova et 
al. (2007) that the comets characterized by a strong silicate feature in thermal infrared and high polarization have more porous particles, as it was first pointed out by Li and Greenberg (1998), whereas the comets characterized by a weak silicate feature and low polarization contain more compact particles. This model turned out to account for optical and infrared observations of ejecta from comet 9P/Tempel 1 during Deep Impact mission (Yamamoto et al., 2008). One more advantage of this model is that it is consistent with the data on comet dust composition obtained in situ for comet 1P/Halley and with the structure of Interplanetary Dust Particles (IDPs) (Jessberger et al., 2001). However, as explained later, this model does not provide quantitative fits to the observed dependence of polarization on phase angle. Here, we present a more advanced model that account for all the optical properties of comet dust not only qualitatively but also quantitatively.

\section{Ground-based Observations and Their Inter- pretation}

Despite the difference in size, age, orbit, and source of origin, the vast majority of comets shows very similar photopolarimetric characteristics in the visible (see Kolokolova et al., 2004a, b, and references therein). They are the following:

- Low geometric albedo of the particles, close to 4-5\%.

- Prominent forward-scattering and gentle back scattering peaks in the angular dependence of intensity, flat behavior at medium phase angles.

- For a broad range of wavelengths angular dependence of linear polarization demonstrates (i) negative branch of polarization for phase angles $\leq 20^{\circ}$ with the minimum $P_{\min } \approx 2 \%$; ii) bell-shaped positive branch with low maximum of value $P_{\max } \approx 15-25 \%$ at the phase angle in the range $90-100^{\circ}$.

- Usually red or neutral color for a broad range of wavelengths that does not change with the phase angle.

- Polarization at a given phase angle above $30^{\circ}$ usually increases with the wavelength (red polarimetric color); this increase gets larger with the phase angle.

As it was mentioned in Introduction, all of these observational features could be qualitatively simulated if we consider the comet particles as aggregates of submicron grains. The model works well for both porous (Ballistic ClusterCluster Aggregates, BCCA) and more compact (Ballistic Particle-Cluster Aggregates, BPCA) particles.

Using the T-matrix multisphere code (Mackowski and Mishchenko, 1996) Kimura et al. (2006) accomplished a survey of light-scattering properties of aggregates. The best fit to the comet observational data was achieved for the case of the aggregates of $\approx 0.2$ micron-size particles made of a mixture of organics, carbon, silicates with a small admixture of iron sulfide or iron. The last component was added based on detection of FeNi metals in IDPs of likely cometary origin and carbonaceous meteorites (Bradley et al., 1996; Bernatowicz et al., 1999). The presence of FeNi metal in cometary dust is confirmed by the results of the Stardust mission (Zolensky et al., 2006). Such a model could also reproduce a low albedo equal to $4-5 \%$ that is close to the one found from the numerous observations and space mission studies. However, a quantitative fit to the observed degree of polarization seemed to require very big, made of hundreds of thousand monomers, aggregates. In other case the computational results showed too small values of the negative polarization and too high degree of the maximum polarization. Such huge aggregates require enormous computer time and memory to calculate their lightscattering characteristics. Also, domination of large fluffy aggregates in comet dust may be unrealistic assumption at large distances from the nucleus where fragile aggregates get smaller due to fragmentation. Thus, the model definitely requires some improvements which we attempt to provide in this paper based on additional information obtained from the space-mission data.

\section{Comet Dust as Viewed by Space Missions}

The model of aggregates described in the previous section was not developed in isolation from the results of the space mission to comets. First, the aggregate model of comet dust partly was stimulated by studies of IDPs captured in the upper layers of the Earth's atmosphere. These studies showed that IDPs of presumably cometary origin are aggregates of submicron particles (Brownlee et al., 1980). Second, Kimura et al. (2003) used the composition of comet dust consistent with the average composition of the dust in comet 1P/Halley measured in situ by Giotto mission (Jessberger et al., 1988). Hereafter we will call this composition Halley-like. In the next section we describe it in more detail.

Recent space missions have added new information to the known properties of the comet dust. The most significant insight was provided by the Stardust mission. This is not surprising since this was the mission specifically designed to study the comet dust in situ and to bring back samples of comet dust. The in-situ measurements by instruments DFMI and CIDA allowed revealing the size distribution of the particles (Green et al., 2004; see in more detail below) and provided some information on the composition of the dust (Kissel et al., 2004). However, the most important findings have been done during the study of the returned samples of the comet dust.

The returned samples present the comet dust particles captured when a special panel made of aerogel cells was exposed to the dust flux in the coma. Study of the tracks of the dust particles in the aerogel and in the aluminum foil around the cells revealed the structure of the particles. The majority of them appeared to be fragile aggregates of small submicron particles. Their composition was complex and included both silicate and carbonaceous materials as this was found for comet $1 \mathrm{P} / \mathrm{Halley}$. However, besides the aggregates, rather large non-agglomerated, solid silicate particles were also found among the Stardust returned samples (see, e.g., Flynn, 2008). In-situ study of comet $1 \mathrm{P} /$ Halley showed that dust in comet $1 \mathrm{P} /$ Halley consisted of three types of particles (Fomenkova, 1999): rock (silicate), CHON (organic) and mixed (averaged, Halleylike dust composition) particles. The rock particles were shown to be solid whereas mixed and organic were fluffy (Jessberger and Kissel, 1991). Both rock and mixed parti- 
cles were found in the Stardust returned samples. The absence of pure organic particles in Stardust samples is not surprising. It is very likely that the high-speed interaction of the particles with the aerogel caused evaporation of small organic particles. Notice that organic-rich particles were detected at in-situ measurements by Stardust CIDA instrument (Kissel et al., 2004).

In the next section we consider a model of comet dust that includes the space-mission results discussed in this section. Besides the fact that the model considers the comet dust as a mixture of solid silicate and aggregated organic and Halleylike particles, the particle size distribution should be also consistent with the one found by cometary space missions.

There have been several sets of space-mission data that allowed determining size distribution of comet particles. Hörz et al. (2006) summarizes the comet dust size distribution obtained from the Stardust studies of tracks in aerogel and craters in the aluminum foil, Stardust DFMI insitu measurements of comet 81P/Wild 2 dust, and Giotto DIDSY and PIA measurements of dust in comet 1P/Halley. All size distributions look very much alike and in all cases consist of three power-law size distributions: least steep for the particles smaller than 1 micron, steeper for the particles of size between 1 and 100 micron and even steeper for the larger particles. The exponent of the power law for all the cases is negative and changes within 2-4 that is close to the values obtained from ground based observations, e.g., observations of comets C/1996 C1 Tabur and 174P/Exhclus (Kolokolova et al., 2001; Bauer et al., 2008).

\section{Combined Model of Comet Dust}

The combined model of comet dust outlined in the previous section is combined in two meanings. First, it combines the results of successful interpretation of ground-based observations with the in-situ space mission data and laboratory studies of the returned samples of comet dust. Second, it combines particles of two structural types (aggregates and solid particles) and three types of composition (silicate particles, organic particles, and particles of Halley-like composition).

We consider light-scattering by the solid silicate particles using the T-matrix method for spheroids by Mishchenko et al. (1996). The refractive index equal to $1.6+0.001 i$ that we use in the calculations is close to the values of optical constants for glass of olivine or pyroxene composition reported by Dorshchner et al. (1995). The particles are presented by multishaped, polydisperse mixture of spheroids. Size distribution of the particles is selected to be consistent with the results of studies of comet dust by the space missions described at the end of the previous section. The power of the size distribution of the dust particles has been found negative in a range of the values from 2 to 4 and the exact value depends not only on the size of particles but also on the method used to study the size distribution and on the position of the instruments in the coma. This is why we decided not to follow the found size distributions in detail but consider a single power law with the power equal to -3 , which represents the average value among the found ones. Multishaped properties of the ensemble were simulated by considering a mixture of oblate and prolate spheroids with the axis ratio within the range 1-2.5.

Aggregates in the combined model were represented as Ballistic-Cluster-Cluster Aggregates (BCCA) of organic and Halley-like composition. Aggregates were made of 256 monomers of radius $0.1 \mu \mathrm{m}$. The light-scattering properties of these aggregates were calculated using the multisphere T-matrix code by Mackowski and Mishchenko (1996). In case of organic aggregates we use the refractive indices from Li and Greenberg (1997). The Halley-like composition, as it was mentioned above, represents average composition found at in-situ measurements of the dust in comet 1P/Halley (Jessberger et al., 1988). It represents a mixture of silicates, metals, and carbonaceous materials. We suppose that one third of the carbonaceous materials are in the form of organic refractory and two thirds are in the form of amorphous carbon. This is based on a comparison of the nitrogen abundance between Halley's and interstellar dust (Kimura et al., 2003). We consider that silicates, iron, and organics refractory are embedded in amorphous carbon and estimate the average refractive index of the mixture using the multi-component Maxwell Garnett mixing rule (Bohren and Huffman, 1983, chapter 8.5). Taking into account the elemental abundances of Halley's dust the volume filling factors are $31.76 \%$ for silicates, $2.56 \%$ for iron, and $65.68 \%$ for carbonaceous materials. The refractive indices of iron, organics refractory, and amorphous carbon are taken from Johnson and Christy (1974), Li and Greenberg (1997), and Rouleau and Martin (1991), respectively. For silicates we adapt the refractive index of astronomical silicate from Laor and Draine (1993). This material has slightly higher absorption than such silicates as olivine and pyroxene that allows us to simulate a slight admixture of iron sulfide found in the comet dust (Zolensky et al., 2006; Flynn, 2008).

We computed the intensity in the scattered plane $I_{\text {par }}$ and in the perpendicular plane $I_{\text {per }}$ separately for polydisperse multishaped spheroids, for organic BCCA, and for Halley-like BCCA, and then calculated the final intensity $I=I_{\mathrm{per}}+I_{\mathrm{par}}$ and polarization $P=\left(I_{\mathrm{per}}-I_{\mathrm{par}}\right) / I$ summarizing $I_{\mathrm{per}}$ and $I_{\mathrm{par}}$ for all constituents of the mixture with some factors that were varied to control the silicateto-carbonaceous-materials ratio and to adjust our results to the observational data.

The best fit results are shown in Fig. 1 where the top picture shows the change of albedo at two wavelengths, 0.45 and $0.6 \mu \mathrm{m}$, and the bottom one shows the phase-angle change in polarization. One can see that we have managed to fit the cometary photopolarimetric data rather well. The brightness follows the phase angular dependence described in Section 2, albedo at zero phase angle is small, about $3 \%$ for the red filter, and the brightness at the shorter wavelength is smaller than at the longer one, i.e., the color is red. The calculated polarization also fits the angular dependence known from the observations of comets, and not only qualitatively but also quantitatively. Even though we get a deeper negative polarization branch than was observed, with the minimum $\leq 4 \%$, it changes the sign at approximately $25^{\circ}$, and reaches the maximum values at about $25-30 \%$ at the phase angle around $95^{\circ}$ that fits very well to the observational data. Also, the polarization at the shorter wavelength is smaller than at the longer one (red polarimetric color) and 

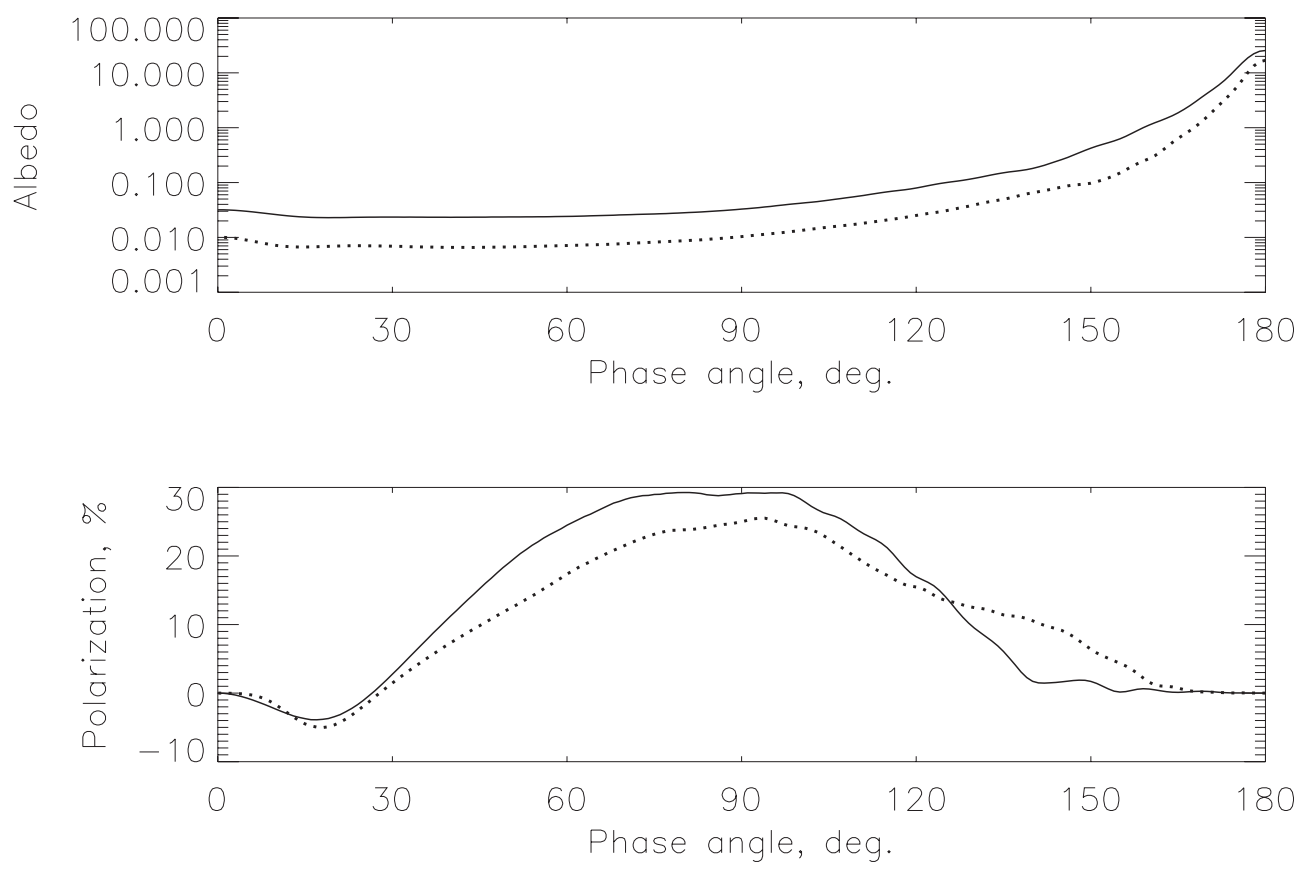

Fig. 1. Dependence of albedo (top) and polarization (bottom) on the phase angle for the mixture of the aggregates of organic and Halley-like composition and compact silicate particles described in Section 4. Solid line is for the wavelength $0.6 \mu \mathrm{m}$ and dotted line is for the wavelength $0.45 \mu \mathrm{m}$.

the difference between them increase with the phase angle that correctly reproduces the observational trends.

As we have mentioned above, we have one more parameter that was controlled during our calculations. This is the mass ratio of silicate to carbonaceous materials. The result presented at Fig. 1 corresponds to this ratio equal to unity that is consistent with the elemental abundance of the comet Halley dust (Jessberger et al., 1988). Finally, the best-fit result appears to be in accordance with the abundance of rock, organic, and mixed particles found at in-situ measurements of the dust in comet 1P/Halley. We found that the best fit is achieved if the ratio of rock particles to the organic ones and to the Halley-like aggregates is equal to $0.30: 0.44: 0.26$ that is consistent with the results by Fomenkova (1999) who showed that the rock particles represented $1 / 3$ of the Halley dust. Our model also agrees with the fact that the rock particles in the Halley dust were compact whereas other particles were fluffy (Jessberger and Kissel, 1991).

The presented combined model shows a significant improvement in fitting the whole scope of the ground-based and space-mission data for comet dust. The further development of the model (e.g., by including larger size of particles and larger axes ratio for compact grains) is planned to get a better quantitative fit to the polarization and albedo values.

\section{Conclusions}

The model of comet dust that combines two types of particles: aggregates of submicron particles of organic and Halley-like composition and compact silicate particles can correctly reproduce all observational data and fits to the data on albedo, photometric phase curve, polarimetric phase curve, color, polarimetric color, and ratio of silicates and organics in the comet dust. It confirms the results obtained in situ for the dust in comet $1 \mathrm{P} /$ Halley and is consistent with recent Stardust findings. This model could provide a much better fit to the photometric and polarimetric observational data than the model that considered only aggregates. The model correctly reproduces the polarimetric data, including a pronounced negative polarization at small phase angles and the positive polarization with the maximum value less than $30 \%$ at the phase angle around $95^{\circ}$ and red polarimetric color. It also shows red color of comet dust, albedo of the dust equal to $\approx 3 \%$, and the mass ratio of silicate to carbonaceous materials in the dust equal to unity that is in accordance with the elemental abundances of Halley's dust. Finally, it shows the ratio of rock, organic, and mixed particles in the dust that agrees with the Giotto in-situ measurements.

Acknowledgments. This work is in part supported by the Japanese Ministry of Education, Culture, Sports, Science, and Technology MEXT (Monbu Kagaku Sho) under Grant-in-Aid for Scientific Research on Priority Areas.

\section{References}

Bauer, J. M., Y.-J. Choi, P. R. Weissman, J. A. Stansberry, Y. R. Fernandez, H. G. Roe, B. J. Buratti, and Hyun-Il Sung, The large-grained dust coma of 174P/Echeclus, Publ. Astron. Soc. Pacific, 120, 393-404, 2008.

Bernatowicz, T., J. Bradley, S. Amari, S. Messenger, and R. Lewis, New kinds of massive star condensates in a presolar graphite from Murchison, Lunar Planet. Sci., 30, 1392-1393, 1999.

Bohren, C. F. and D. R. Huffman, Absorption and Scattering of Light by Small Particles, Wiley-Interscience, New York, 1983.

Bradley, J. P. and T. Ireland, The search for interstellar components in interplanetary dust particles, in IAU Colloq. 150, Physics, Chemistry, and Dynamics of Interplanetary Dust, edited by B. A. S. Gustafson and M. S. Hanner, 275-283, ASP Conf. Ser. 104, San Francisco: ASP, 1996. Brownlee, D. E., L. Pilachowski, E. Olszewski, and P. W. Hodge, in Solid Particles in the Solar System, edited by I. Halliday, and B. A. McIntosh, 333-342, D. Reidel, Dordrecht, 1980.

Dorshchner, J., B. Begermann, T. Henning, C. Jaeger, and H. Mutshchke, Steps toward interstellar silicate mineralogy. II. Study of $\mathrm{Mg}$-Fe-silicate glasses of variable composition, Astron. Astrophys., 300, 503-517, 
1995.

Flynn, G. J., Physical, chemical, and mineralogical properties of comet 81P/Wild 2 particles collected by Stardust, Earth Moon Planets, 102, 447-459, 2008.

Fomenkova, M., On the organic refractory component of cometary dust, Space Sci. Rev., 90, 109-114, 1999.

Green, S. F., J. A. M. McDonnell, N. McBride, M. T. S. H. Colwell, A. J. Tuzzolino, T. E. Economou, P. Tsou, B. C. Clark, and D. E. Brownlee, The dust mass distribution of comet 81P/Wild 2, J. Geophys. Res., 109, E12S04, 2004.

Hörz, F. and 44 coauthors, Impact features on Stardust: Implications for comet 81P/Wild 2 dust, Science, 314, 1716-1719, 2006.

Jessberger, E. K. and J. Kissel, Chemical properties of cometary dust and a note on carbon isotopes, in Comets in the Post-Halley Era, v.2, 10751092, 1991.

Jessberger, E. K., A. Christoforidis, and J. Kissel, Aspects of the major element composition of Halley's dust, Nature, 332(6166), 691-695, 1988.

Jessberger, E. K., T. Stephan, D. Rost et al., in Interplanetary Dust, edited by E. Grün, B. A. S. Gustafson, S. F. Dermott, and H. Fechtig, 253-294, Springer-Verlag, Heidelberg, 2001

Johnson, P. B. and R. W. Christy, Optical constants of metals: Ti, V, Cr, $\mathrm{Mn}, \mathrm{Fe}, \mathrm{Co}, \mathrm{Ni}$, and Pd, Phys. Rev. B, 9, 5056-5070, 1974.

Kimura, H., L. Kolokolova, and I. Mann, Optical properties of cometary dust: Constraints from numerical studies on light scattering by aggregate particles, Astron. Astrophys., 407, L5-L8, 2003.

Kimura, H., L. Kolokolova, and I. Mann, Light scattering by cometary dust numerically simulated with aggregate particles consisting of identical spheres, Astron. Astrophys., 449, 1243-1254, 2006.

Kissel, J., F. R. Krueger, J. Siln, and B. C. Clark, The cometary and interstellar dust analyzer at comet 81P/Wild 2, Science, 304, 17741776, 2004.

Kolokolova, L., L. M. Lara, R. Schulz, J. A. Stwe, and G. P. Tozzi, Properties and evolution of dust in comet Tabur (C/1996 Q1) from the color maps, Icarus, 153, 197-207, 2001.

Kolokolova, L., M. Hanner, A.-Ch. Levasseur-Regourd, and B. A. S. Gustafson, Physical properties of cometary dust from light scattering and emission, in Comets II, edited by Festou, M. C., H. U. Keller, and
H. A. Weaver, 577-604, University of Arizona Press, 2004a.

Kolokolova, L., H. Kimura, and I. Mann, Characterization of dust particles using photopolarimetric data: Example of cometary dust, in Photopolarimetry in Remote Sensing, edited by G. Videen, Ya. Yatskiv, and M. Mishchenko, 431-454, Kluwer Academic Publishers, Dordrecht, The Netherlands, $2004 \mathrm{~b}$.

Kolokolova, L., H. Kimura, N. Kiselev, and V. Rosenbush, Polarimetric and infrared evidence of two types of dust in comets, Astron. Astrophys., 463, 1189-1196, 2007.

Laor, A. and B. T. Draine, Spectroscopic constraints on the properties of dust in active galactic nuclei, Astrophys. J., 402, 441-468, 1993.

Li, A. and J. M. Greenberg, A unified model of interstellar dust, Astron. Astrophys., 323, 566-584, 1997.

Li, A. and J. M. Greenberg, From interstellar dust to comets: Infrared emission from comet Hale-Bopp (C/1995 O1), Astrophys. J., 498, L83L86, 1998.

Mackowski, D. W. and M. I. Mishchenko, Calculation of the T matrix and the scattering matrix for ensembles of spheres, J. Opt. Soc. Am. A, 13, 2266-2278, 1996.

Mann, I., H. Kimura, and L. Kolokolova, A comprehensive model to describe light scattering properties of cometary dust, J. Quant. Spectrosc. Radiat. Transfer, 89, 291-301, 2004.

Mishchenko, M. I., L. D. Travis, and D. W. Mackowski, T-matrix computations of light scattering by nonspherical particles: a review, J. Quant. Spectrosc. Radiat. Transfer, 55, 535-575, 1996.

Mukai, T., H. Ishimoto, T. Kozasa, J. Blum, and J. M. Greenberg, Radiation pressure forces of fluffy porous grains, Astron. Astrophys., 262, 315320, 1992.

Rouleau, F. and P. G. Martin, Shape and clustering effects on the optical properties of amorphous carbon, Astrophys. J., 377, 526-540, 1991.

Yamamoto, S., H. Kimura, E. Zubko, H. Kobayashi, K. Wada, M. Ishiguro, and T. Matsui, Comet 9P/Tempel 1: Interpretation with the Deep Impact results, Astrophys. J., 673, L199-L202, 2008.

Zolensky, M. E. and 75 co-authors, Mineralogy and petrology of comet 81P/Wild 2 nucleus samples, Science, 314, 1735-1737, 2006.

L. Kolokolova (e-mail: ludmilla@astro.umd.edu) and H. Kimura 